\title{
Nonuniversal correlations in multiple scattering
}

\author{
S. E. Skipetrov and R. Maynard \\ Laboratoire de Physique et Modélisation des Milieux Condensés, Université Joseph Fourier, \\ Maison des Magistères - CNRS, B.P. 166, 38042 Grenoble Cedex 9, France
}

(October 30, 2018)

\begin{abstract}
We show that intensity of a wave created by a source embedded inside a three-dimensional disordered medium exhibits a non-universal space-time correlation which depends explicitly on the short-distance properties of disorder, source size, and dynamics of disorder in the immediate neighborhood of the source. This correlation has an infinite spatial range and is long-ranged in time. We suggest that a technique of "diffuse microscopy" might be developed employing spatially-selective sensitivity of the considered correlation to the disorder properties.

42.25.Dd, 71.55.Jv
\end{abstract}

\section{INTRODUCTION}

Tremendous progress has been made during the last years in the understanding of multiple scattering of waves in disordered media in so-called mesoscopic regime. 1 This regime is commonly defined by

$$
\lambda \ll \ell \ll L \ll L_{a b s},
$$

where $\lambda$ is the wavelength, $\ell$ is the mean free path, $L$ is the sample size (or source-detector separation in the case of infinite medium), and $L_{a b s}$ is the absorption length. In particular, considerable attention has been paid to the study of the correlation function of intensity fluctuations

$$
C(\mathbf{r}, \Delta \mathbf{r}, \tau)=\frac{\langle\delta I(\mathbf{r}, t) \delta I(\mathbf{r}+\Delta \mathbf{r}, t+\tau)\rangle}{\langle I(\mathbf{r}, t)\rangle\langle I(\mathbf{r}+\Delta \mathbf{r}, t)\rangle}
$$


where $I(\mathbf{r}, t)=|\psi(\mathbf{r}, t)|^{2}$ is the intensity of the wave field $\psi(\mathbf{r}, t), \delta I(\mathbf{r}, t)=I(\mathbf{r}, t)-\langle I(\mathbf{r}, t)\rangle$, angular brackets $\langle\cdots\rangle$ denote ensemble averaging, and the random fields $\psi(\mathbf{r}, t), I(\mathbf{r}, t)$ are assumed to be stationary. In the present paper, we are mainly interested in the correlation of intensities in two distant points separated by a distance superior to the mean free path: $\Delta r>\ell$.

Several contributions to $C$ can be identified. correlations arise from interference between the waves arriving at the points $\mathbf{r}$ and $\mathbf{r}+$ $\Delta \mathbf{r}$. Weak long-range correlations are due to the interaction of diffusing modes in the medium. Finally, tiny infinite-range correlations manifest themselves in the phenomenon of universal conductance fluctuations, 1 and have been recently observed for optical waves. listed contributions to the correlation function are commonly denoted by $C_{1}, C_{2}$ and $C_{3}$, respectively. It is important to emphasize that the magnitudes of these correlations scale with some dimensionless parameter and are not sensitive to the short-distance properties of disorder provided that $\left|\mathbf{r}-\mathbf{r}_{0}\right|,\left|\mathbf{r}+\Delta \mathbf{r}-\mathbf{r}_{0}\right|>\ell$. For the case of plane wave transmission through a slab, such a parameter is the dimensionless conductance $g \sim N \ell / L$, where $N \sim$ $k_{0}^{2} A$ is the number of transport channels, $k_{0}=2 \pi / \lambda$ is the wavenumber, and $A$ is the area of the slab surface. For $g \gg 1$, which is a typical experimental situation, one finds 目 $C_{1} \sim 1$, $C_{2} \sim g^{-1}, C_{3} \sim g^{-2}$. For a medium of infinite extent, the relevant parameter is the product $k_{0} \ell: C_{1} \sim 1, C_{2} \sim\left(k_{0} \ell\right)^{-2}, C_{3} \sim\left(k_{0} \ell\right)^{-4}$, where $k_{0} \ell \gg 1$ is assumed. In all cases, the only medium-specific quantity determining the magnitude of the correlation is the mean free path $\ell$, and thus we call the $C_{1}, C_{2}$, and $C_{3}$ correlations universal as they are independent of the particular features of disorder, and only depend on the global parameter $\ell$.

Recently, a new contribution to the intensity correlation in random media $\left(C_{0}\right)$ has been conjectured 6 This contribution originates from the scattering of wave near its source. In the case of a point source embedded in an infinite three-dimensional medium with fluctuations of the dielectric constant obeying white-noise Gaussian statistics, for $\Delta r>\ell$ and $\tau=0$, it has been found 6 


$$
C_{0}(\mathbf{r}, \Delta \mathbf{r}, 0)=\frac{\pi}{k_{0} \ell}
$$

The physical process giving rise to the $C_{0}$ correlation consists in the scattering of the wave in the immediate neighborhood of its source (more precisely, at a distance of order $\lambda$ from the source), which produces a secondary wave propagating to the distant points $\mathbf{r}$ and $\mathbf{r}+\Delta \mathbf{r}$ by diffusion. It turns out that the secondary waves created in such a way are correlated, and hence the described physical process establishes correlation between intensities at different points of the medium. It is important to emphasize that this correlation has an infinite spatial range.

A fundamental difference between $C_{0}$ and other types of correlation is that $C_{0}$ should be sensitive to the short-distance details of disorder (e.g., to its correlation length) 1 Moreover, as this contribution to the correlation function is due to the scattering in the vicinity of the source, one could expect that $C_{0}$ should depend on the geometrical parameters of the source and, in particular, on its size. Finally, for moving scatterers and $\tau \neq 0, C_{0}$ should also be sensitive to the dynamics of disordered medium in the immediate neighborhood of the source. It follows then that the $C_{0}$ contribution to the correlation function is non-universal.

In the present paper, we show that the $C_{0}$ correlation function does exhibit a strongly non-universal behavior. First, we study the dependence of $C_{0}$ on the correlation length of disorder $\ell_{\varepsilon}$. It is shown that the $C_{0}$ correlation function depends explicitly on $k_{0} \ell_{\varepsilon}$, and, more precisely, that it decreases linearly with $k_{0} \ell_{\varepsilon}$ for $k_{0} \ell_{\varepsilon} \ll 1$. The sensitivity of the $C_{0}$ correlation function to the correlation length of disorder might be used for sensitive specific measurements of $\ell_{\varepsilon}$. Next, we study the effect of the source size $a$ on the $C_{0}$ correlation. We show that $C_{0}$ decreases as $a$ is increased. The decrease of $C_{0}$ with $a$ is linear for $k_{0} a \ll 1$ and becomes power-law $\left[C_{0} \propto\left(k_{0} a\right)^{-1}\right]$ for $k_{0} a \gg 1$. Finally, we compute the space-time intensity correlation function $C_{0}(\mathbf{r}, \Delta \mathbf{r}, \tau)$. We show that this correlation function is sensitive to the motion of scatterers in the immediate neighborhood of the source. In the case of disordered medium consisting of scatterers undergoing Brownian motion, $C_{0}$ decays linearly with $\sqrt{\tau / \tau_{0}}$ as $\tau \ll \tau_{0}$ and exhibits a power-law behavior $\left[C_{0} \propto\left(\tau / \tau_{0}\right)^{-3 / 2}\right]$ for $\tau \gg \tau_{0}$, with $\tau_{0}$ being a 
single-scattering correlation time. The decay of $C_{0}$ with time is exclusively determined by the motion of scatterers located at a distance of order $\lambda$ from the source, and is not sensitive to the dynamics of the rest of the medium. Measurements of $C_{0}$ could therefore provide a sensitive spatially-selective probe of scatterer motion in disordered media.

\section{SPATIAL CORRELATION}

Following Ref. 6, we consider an infinite three-dimensional disordered medium with a monochromatic source of scalar waves located at $\mathbf{r}_{0}$. We assume that the source is spherically symmetric and has a radius $a$. To treat the problem of non-zero $a$, one needs to specify the boundary conditions for the wave field at the surface $\left|\mathbf{r}-\mathbf{r}_{0}\right|=a$. We assume that waves are

transmitted through the region of space occupied by the source without being scattered 7 Then for $\left|\mathbf{r}-\mathbf{r}_{0}\right|>a$ the wave field $\psi(\mathbf{r}, t)$ obeys the scalar wave equation with a point source at $\mathbf{r}_{0}$ :

$$
\left\{\nabla^{2}+k_{0}^{2}[1+\delta \varepsilon(\mathbf{r}, t)+i \eta]\right\} \psi(\mathbf{r}, t)=\delta\left(\mathbf{r}-\mathbf{r}_{0}\right)
$$

where $\delta \varepsilon(\mathbf{r}, t)$ is the fluctuating part of the dielectric constant $\left[\delta \varepsilon(\mathbf{r}, t) \equiv 0\right.$ for $\left.\left|\mathbf{r}-\mathbf{r}_{0}\right|<a\right]$, and $\eta$ is a positive infinitesimal ensuring the uniqueness of solution of Eq. (4). The wave equation can be written in the form of Eq. (4) only if $\delta \varepsilon(\mathbf{r}, t)$ does not change significantly at the time scale of $\left(c k_{0}\right)^{-1}$, with $c$ being the speed of propagation in the average medium: $\partial / \partial t[\delta \varepsilon(\mathbf{r}, t)] \ll c k_{0}$.

Let us first consider the purely spatial correlation function $C(\mathbf{r}, \Delta \mathbf{r}, 0)$. The time evolution of $\delta \varepsilon(\mathbf{r}, t)$ is of no importance in this case, and the statistical properties of $\delta \varepsilon(\mathbf{r}, t)$ are completely specified by its average value and covariance. Without loss of generality, we consider $\delta \varepsilon(\mathbf{r}, t)$ which obeys Gaussian statistics with zero average and correlation length $\ell_{\varepsilon}$ :

$$
\begin{aligned}
& \langle\delta \varepsilon(\mathbf{r}, t)\rangle=0, \\
& B_{\varepsilon}(\Delta \mathbf{r})=\langle\delta \varepsilon(\mathbf{r}, t) \delta \varepsilon(\mathbf{r}+\Delta \mathbf{r}, t)\rangle=\sigma_{\varepsilon}^{2} \exp \left(-\frac{\Delta r^{2}}{\ell_{\varepsilon}^{2}}\right),
\end{aligned}
$$


where $\left|\mathbf{r}-\mathbf{r}_{0}\right|,\left|\mathbf{r}+\Delta \mathbf{r}-\mathbf{r}_{0}\right|>a$. In the present paper we restrict ourselves to weak scattering $\left(k_{0}^{2} \ell_{\varepsilon}^{2} \sigma_{\varepsilon}^{2} \ll 1\right)$ and short correlation length $\left(\ell_{\varepsilon} \ll \lambda\right)$. The first assumption allows us to benefit from a standard diagrammatic techniques 8 in the first order Born approximation, while the second one permits to not distinguish between the scattering and the transport mean free paths. The case of $\ell_{\varepsilon} \gtrsim \lambda$ calls for a more sophisticated treatment which is beyond the scope of this paper. For $k_{0}^{2} \ell_{\varepsilon}^{2} \sigma_{\varepsilon}^{2} \ll 1$ and $\ell_{\varepsilon} \ll \lambda$, the mean free path is $\ell=4 \pi /\left(u k_{0}^{4}\right)$ with $u=\pi^{3 / 2} \sigma_{\varepsilon}^{2} \ell_{\varepsilon}^{3}$. In the following we assume that any variation of $\ell_{\varepsilon}$ implies corresponding change of $\sigma_{\varepsilon}$ so that $\ell$ remains unchanged.

As shown in Ref. 6, in a weak scattering limit $\left(k_{0} \ell \gg 1\right)$, the leading contribution to $\langle\delta I(\mathbf{r}, t) \delta I(\mathbf{r}+\Delta \mathbf{r}, t+\tau)\rangle$ can be represented by a diagram depicted in Fig. 1, where the ladder propagators are $L\left(\mathbf{r}_{1}, \mathbf{r}_{2}\right)=3 /\left(\ell^{3}\left|\mathbf{r}_{1}-\mathbf{r}_{2}\right|\right)$, and $V$ is a vertex to be calculated with account for the short-distance properties of disorder and the size of the source. This vertex is a short-range object and can be replaced by a number. We show the leading contributions to $V$ in Fig. 2. Note that the diagrams (c) and (d) contribute to the vertex only if $\ell_{\varepsilon} \neq 0$ or $a \neq 0$ and therefore they have not been counted in Ref. 6, where only the case of $\ell_{\varepsilon}=a=0$ has been considered.

The calculation of the diagrams of Fig. 2 is performed similarly to that of Ref. 6. For the diagram (b) of Fig. 2 we obtain, for instance,

$$
V^{(b)}=k_{0}^{4}\left(\frac{\ell}{4 \pi}\right)^{2} \int d^{3} \mathbf{r}_{1} \int d^{3} \mathbf{r}_{4} \bar{G}\left(\mathbf{r}_{1}-\mathbf{r}_{0}\right) \bar{G}^{*}\left(\mathbf{r}_{4}-\mathbf{r}_{0}\right) f^{*}\left(\mathbf{r}_{1}-\mathbf{r}_{0}\right) f\left(\mathbf{r}_{4}-\mathbf{r}_{0}\right) B_{\varepsilon}\left(\mathbf{r}_{1}, \mathbf{r}_{4}\right),
$$

where integrations cover the whole space except the interior of a sphere of radius $a$ centered at $\mathbf{r}_{0}$,

$$
\bar{G}\left(\mathbf{r}-\mathbf{r}_{0}\right)=-\frac{1}{4 \pi\left|\mathbf{r}-\mathbf{r}_{0}\right|} \exp \left[\left(i k_{0}-\frac{1}{2 \ell}\right)\left|\mathbf{r}-\mathbf{r}_{0}\right|\right]
$$

is the average Green function of Eq. (四), and

$$
f\left(\mathbf{r}-\mathbf{r}_{0}\right)=\frac{\sin \left(k_{0}\left|\mathbf{r}-\mathbf{r}_{0}\right|\right)}{k_{0}\left|\mathbf{r}-\mathbf{r}_{0}\right|} \exp \left(-\frac{\left|\mathbf{r}-\mathbf{r}_{0}\right|}{2 \ell}\right) .
$$

The derivation of the two above expressions is performed under important assumptions of $k_{0} \ell \gg$ and $a \ll \ell$. The second assumption allows us to neglect the absence of the dielectric 
constant fluctuations inside the region of space occupied by the source, which simplifies greatly the derivations.

Results similar to Eq. (7) are also obtained for the three other diagrams of Fig. 2. The diagram (c), for example, is given by the same integral as in the Eq. (7) with the integrand $\bar{G}^{*}\left(\mathbf{r}_{1}-\mathbf{r}_{0}\right) \bar{G}^{*}\left(\mathbf{r}_{4}-\mathbf{r}_{0}\right) f\left(\mathbf{r}_{1}-\mathbf{r}_{0}\right) f\left(\mathbf{r}_{4}-\mathbf{r}_{0}\right) B_{\varepsilon}\left(\mathbf{r}_{1}, \mathbf{r}_{4}\right)$. Next, diagrams (a) and (d) are obtained from (b) and (c) by a complex conjugation. Finally, taking the sum of all the four diagrams, performing angular integrals, and introducing $x_{1}=k_{0} r_{1}$ and $x_{2}=k_{0} r_{4}$ as new variables of integration, we get

$$
V=V^{(a)}+\ldots+V^{(d)}=\frac{\ell}{16 \pi k_{0}} F\left(k_{0} \ell_{\varepsilon}, k_{0} a\right)
$$

where

$$
\begin{aligned}
F(\alpha, \beta) & =\frac{1}{\pi^{3 / 2}} \frac{1}{\alpha} \int_{\beta}^{\infty} d x_{1} \int_{\beta}^{\infty} d x_{2} \frac{\sin \left(2 x_{1}\right)}{x_{1}} \frac{\sin \left(2 x_{2}\right)}{x_{2}} \\
& \times\left\{\exp \left[-\left(\frac{x_{1}-x_{2}}{\alpha}\right)^{2}\right]-\exp \left[-\left(\frac{x_{1}+x_{2}}{\alpha}\right)^{2}\right]\right\} .
\end{aligned}
$$

The rest of the calculation is straightforward. The diagram of Fig. 1 yields $\langle\delta I(\mathbf{r}, t) \delta I(\mathbf{r}+\Delta \mathbf{r}, t+\tau)\rangle=V L\left(\mathbf{r}_{0}, \mathbf{r}\right) L\left(\mathbf{r}_{0}, \mathbf{r}+\Delta \mathbf{r}\right)[\ell /(4 \pi)]^{2}$, where the factor $[\ell /(4 \pi)]^{2}$ accounts for the two vertices connecting the diffusion ladders to the points $\mathbf{r}$ and $\mathbf{r}+\Delta \mathbf{r}$. As the average intensity is $\langle I(\mathbf{r}, t)\rangle=[\ell /(4 \pi)]^{2} L\left(\mathbf{r}_{0}, \mathbf{r}\right)$, $\mathrm{g}$ the contribution of the diagram of Fig. 11 to the correlation function defined by Eq. (2) becomes

$$
C_{0}(\mathbf{r}, \Delta \mathbf{r}, 0)=\frac{\pi}{k_{0} \ell} F\left(k_{0} \ell_{\varepsilon}, k_{0} a\right)
$$

The result (3) is recovered for $\ell_{\varepsilon}=a=0$, as $F(0,0) \equiv 1$. Hence, the factor $F\left(k_{0} \ell_{\varepsilon}, k_{0} a\right)$ describes the effects of source size $a$ and disorder correlation length $\ell_{\varepsilon}$ on the $C_{0}$ correlation. In general, $F\left(k_{0} \ell_{\varepsilon}, k_{0} a\right)$ is a rather complicated function of its arguments. Below we consider separately the following two cases: $(i) a \ll \ell_{\varepsilon} \ll \lambda$ and $(i i) \ell_{\varepsilon} \ll a, \lambda$.

(i). If $a \ll \ell_{\varepsilon} \ll \lambda$, one can set the lower limits of integrations in Eq. (11) equal to zero. Then Eq. (11) can be simplified, although the simplification requires some care, as the integrand of Eq. (11) does not have finite derivatives at $\alpha=0$ and cannot be therefore 
expanded in a power series near this point. Instead, we represent the expression in the curved brackets of Eq. (11) as $2 \exp \left[-\left(x_{1}^{2}+x_{2}^{2}\right) / \alpha^{2}\right] \sinh \left(2 x_{1} x_{2} / \alpha^{2}\right)$ and then expand $\sinh (\cdots)$ in Taylor series. Carrying out integrations, we obtain 10

$$
F(\alpha, 0)=\frac{2}{\pi} \alpha \sum_{n=0}^{\infty} \frac{\Gamma(n+1)}{\Gamma(n+3 / 2)}{ }_{1} F_{1}^{2}\left(n+1 ; 3 / 2 ;-\alpha^{2}\right),
$$

where ${ }_{1} F_{1}(\cdots)$ is the confluent hypergeometric function. While the series in Eq. (13) converges sufficiently fast for $\alpha>1$, the convergence slows down as $\alpha$ becomes less than unity.

As we consider $\ell_{\varepsilon} \ll \lambda$, we are only interested in $F(\alpha, 0)$ for $\alpha \ll 1$. Although Eq. (13) can be evaluated numerically even for $\alpha<1$, this equation is of little help if one wants to get some qualitative insight into the small- $\alpha$ behavior of $F(\alpha, 0)$. To get such an insight, we introduce $t_{1,2}=x_{1,2} / \alpha$ as new variables of integration in Eq. (11). This yields

$$
\begin{aligned}
F(\alpha, 0) & =\frac{2}{\pi^{3 / 2}} \frac{1}{\alpha} \int_{0}^{\infty} d t_{1} \int_{0}^{\infty} d t_{2} \frac{\sin \left(2 \alpha t_{1}\right)}{t_{1}} \frac{\sin \left(2 \alpha t_{2}\right)}{t_{2}} \\
& \times \exp \left(-t_{1}^{2}-t_{2}^{2}\right) \sinh \left(2 t_{1} t_{2}\right)
\end{aligned}
$$

Assuming small $\alpha$, we replace $\sin \left(2 \alpha t_{2}\right)$ by $2 \alpha t_{2}$ and then perform the integrals over $t_{2}$ and $t_{1}$. Finally, we keep in a final result only the terms linear in $\alpha$. This gives

$$
F(\alpha, 0) \simeq 1-\frac{4}{\pi^{3 / 2}} \alpha, \quad \alpha \ll 1
$$

A plot of $F(\alpha, 0)$ obtained by a numerical evaluation of Eq. (13) is presented in Fig. 3 by a solid line. The decay of $F$ is linear for $\alpha<1$, as predicted by Eq. (15). The part of the plot corresponding to $\alpha>1$ is meaningless for a moment, as we have assumed $\ell_{\varepsilon} \ll \lambda$. This part of the plot will be discussed and interpreted in the next section, where the space-time correlation function is considered.

As follows from Eq. (15) and Fig. 3, the $C_{0}$ correlation decreases with increase of the disorder correlation length $\ell_{\varepsilon}$, and the characteristic scale of this decrease is set by $\lambda$. This is physically clear as $C_{0}$ originates from scattering at a distance of order $\lambda$ from the source, which is only possible if the wave "identifies" its environment as disordered already after a distance of order $\lambda$. Such an identification implies that $\delta \varepsilon(\mathbf{r}, t)$ changes significantly at the 
scale of $\lambda$, or, in other words, that the correlation length of the fluctuations of $\delta \varepsilon(\mathbf{r}, t)$ is shorter than $\lambda$. On the basis of this reasoning, we expect that $C_{0}$ should vanish for $\ell_{\varepsilon}>\lambda$, while for $\ell_{\varepsilon} \sim \lambda$ the behavior of $C_{0}$ may be sensitive to a particular model of disorder. In contrast, the linear initial decay (15) of the $C_{0}$ correlation function with the disorder correlation length $\ell_{\varepsilon}$ seems to be rather general and independent of the model of disorder. We have verified that the linear law (15) holds (with an apparent change of numerical factors) not only for the Gaussian covariance of $\delta \varepsilon(\mathbf{r}, t)$ [Eq. (6)], but also for the exponential one $B_{\varepsilon}(\Delta \mathbf{r})=\sigma_{\varepsilon}^{2} \exp \left(-\Delta r / \ell_{\varepsilon}\right)$, as well as for $B_{\varepsilon}(\Delta \mathbf{r})=\sigma_{\varepsilon}^{2} \Pi\left(\Delta r / \ell_{\varepsilon}\right)$, where $\Pi(x)=1$ for $x \leq 1$ and $\Pi(x)=0$ for $x>1$.

An essential result of our analysis is that the $C_{0}$ correlation function appears to be sensitive to the correlation length $\ell_{\varepsilon}$ of the dielectric function fluctuations, which is a shortdistance parameter of disorder. We remind that all the previously studied types of correlation $\left(C_{1}, C_{2}, C_{3}\right)$ are not sensitive to such details of the scattering medium, and only depend on the mean free path $\ell$. We conclude then that the sensitivity to the short-distance properties of disordered medium is a distinctive feature of the $C_{0}$ correlation function. Measurements of $C_{0}$ could thus provide unambiguous information on the disorder correlation length. It is rather surprising that such information is not lost as a result of multiple scattering, as one would expect, but determines the long-range spatial correlation in the multiple-scattering speckle pattern.

Another important outcome of the presented consideration is that the correlation length $\ell_{\varepsilon}$ entering into Eq. (12) is that characteristic for the disorder in the immediate neighborhood of the source (more precisely, at a distance of order $\lambda$ from it). The information about the rest of the medium is only contained in the mean free path $\ell$. Measurements of the $C_{0}$ correlation could therefore be used for "diffuse microscopy" of disordered media with spatial resolution of order $\lambda$. A possibility of such a microscopy stems from the enhanced sensitivity of the multiple-scattering speckle pattern to the properties of disorder in the vicinity of the source.

(ii). In the case of $\ell_{\varepsilon} \ll a, \lambda$, we can set $\ell_{\varepsilon}=0$ and consider the limit $\alpha \rightarrow 0$ of 
Eq. (11). The exponentials in the curved brackets of Eq. (11) become $\pi^{1 / 2} \alpha \delta\left(x_{1}-x_{2}\right)$ and $\pi^{1 / 2} \alpha \delta\left(x_{1}+x_{2}\right)$, respectively, which yields

$$
\begin{aligned}
F\left(0, k_{0} a\right) & =1-\frac{2}{\pi}\left[\operatorname{Si}\left(4 \mathrm{k}_{0} \mathrm{a}\right)-\frac{\sin ^{2}\left(2 \mathrm{k}_{0} \mathrm{a}\right)}{2 \mathrm{k}_{0} \mathrm{a}}\right] \\
& \simeq \begin{cases}1-(4 / \pi) k_{0} a, & k_{0} a \ll 1, \\
1 /\left(2 \pi k_{0} a\right), & k_{0} a \gg 1,\end{cases}
\end{aligned}
$$

where $\operatorname{Si}(\cdots)$ is the sine integral, and the second line of the equation shows simplified versions of the first one in two limiting cases. Eq. (16) is plotted in Fig. 3 by a dashed line. As $k_{0} a \ll 1$, the decay of $F\left(0, k_{0} a\right)$ is linear, similarly to that of $F\left(k_{0} \ell_{\varepsilon}, 0\right)$ (see the solid line in Fig. 3). When $k_{0} a$ becomes larger than unity, $F\left(0, k_{0} a\right)$ decreases as $\left(k_{0} a\right)^{-1}$. This indicates that although the small size of the source is important for the magnitude of the $C_{0}$ correlation, the correlation process leading to $C_{0}$ survives even for extended $(a \neq 0)$ sources.

The above analysis holds for $\Delta r>\ell$. If $\Delta r<\ell$, an additional contribution to the $C_{0}$ correlation function arises due to scattering in the neighborhood of the observation points. In a complete analogy with the term given by Eq. (12), this second contribution is determined by the short-distance properties of disorder in the vicinity of the observation points $\mathbf{r}$ and $\mathbf{r}+\Delta \mathbf{r}$. For $\Delta r=0$, the contributions owing to scattering near the source and near the observation point become equal and their sum determines a correction to the second moment

of intensity fluctuations $\left\langle\delta I(\mathbf{r}, t)^{2}\right\rangle$. Hence, the most of the conclusions drawn concerning the $C_{0}$ correlation are also true (with obvious insignificant modifications) for $\left\langle\delta I(\mathbf{r}, t)^{2}\right\rangle$.

\section{SPACE-TIME CORRELATION}

We now turn to the case of the space-time correlation function, allowing $\tau \neq 0$ in Eq. (2). It is obvious from general considerations that the correlation function of intensity of a multiple-scattered wave should decrease with $\tau$ once the disordered medium is subject to some non-periodic dynamics. This decrease was first discovered for the $C_{1}$ correlation function,11.12 and recently has been also studied for $C_{2}$ (see Ref. 13) and $C_{3}$ (see Ref. 5). 
As the role of the disorder correlation length $\ell_{\varepsilon}$ has been studied in the previous section, and in order to emphasize the effect of the medium dynamics, we assume $\ell_{\varepsilon}=0$ in the present section. For the sake of concreteness, we consider the disordered medium as a suspension of point-like scatterers which diffuse with a diffusion constant $D_{B}$. The relevant correlation function of the dielectric constant fluctuations is then 2 , 24

$$
B_{\varepsilon}(\Delta \mathbf{r}, \tau)=\langle\delta \varepsilon(\mathbf{r}, t) \delta \varepsilon(\mathbf{r}+\Delta \mathbf{r}, t+\tau)\rangle=\frac{u}{\left(4 \pi D_{B} \tau\right)^{3 / 2}} \exp \left(-\frac{\Delta r^{2}}{4 D_{B} \tau}\right)
$$

Calculation of the diagrams of Figs. 1, 2 is performed very similar to the case of $\tau=0$. We finally obtain

$$
C_{0}(\mathbf{r}, \Delta \mathbf{r}, \tau)=\frac{\pi}{k_{0} \ell} F\left(\sqrt{\tau / \tau_{0}}, k_{0} a\right)
$$

where $F(\cdots)$ is given by Eq. (11), and $\tau_{0}=\left(4 k_{0}^{2} D_{B}\right)^{-1}$ is the single-scattering correlation time.11.12 To simplify the further analysis, we assume $a \ll \lambda,\left(\tau D_{B}\right)^{1 / 2}$ which allows us to extend the lower limits of integrations in Eq. (11) to zero, and to reduce Eq. (11) to Eq. (13).

An approximate expression for $F(\alpha, 0)$ in the limit of small $\alpha$ has been found in the previous section [see Eq. (15)]. To analyze the behavior of $F(\alpha, 0)$ for $\alpha \gg 1$, we take the large- $\alpha$ limit of Eqs. (11), (13):

$$
F(\alpha, 0) \simeq \frac{1}{\pi^{3 / 2}} \frac{1}{\alpha^{3}}, \quad \alpha \gg 1
$$

Hence, the asymptotic behavior of the space-time correlation function can be summarized as follows:

$$
C_{0}(\mathbf{r}, \Delta \mathbf{r}, \tau) \simeq \frac{\pi}{k_{0} \ell} \times \begin{cases}1-\left(4 / \pi^{3 / 2}\right) \sqrt{\tau / \tau_{0}}, & \tau \ll \tau_{0} \\ \left(1 / \pi^{3 / 2}\right)\left(\tau / \tau_{0}\right)^{-3 / 2}, & \tau \gg \tau_{0}\end{cases}
$$

These approximate results are well supported by the numerical evaluation of Eq. (13) which yields the solid line of Fig. 3. Linear initial decay of $F$ (and, consequently, of $C_{0}$ ) with $\sqrt{\tau / \tau_{0}}$ is followed by a power-law long-time tail, as predicted by Eq. (19). 
It is instructive to compare the initial decay of the $C_{0}$ correlation function with that of $C_{1}$, which for the considered case of a point source in an infinite medium and for $\Delta r=0$ reads 12

$$
C_{1}(\mathbf{r}, 0, \tau) \simeq 1-2 \frac{\left|\mathbf{r}-\mathbf{r}_{0}\right|}{\ell} \sqrt{\frac{3 \tau}{2 \tau_{0}}}, \quad \tau \ll \tau_{0}\left(\frac{\ell}{\left|\mathbf{r}-\mathbf{r}_{0}\right|}\right)^{2}
$$

For $\Delta r \neq 0, C_{1}$ decreases exponentially with $\Delta r / \ell$ and thus becomes negligible for $\Delta r>\ell$. Although the functional forms of $C_{0}(\mathbf{r}, \Delta \mathbf{r}, \tau)$ and $C_{1}(\mathbf{r}, 0, \tau)$ for short $\tau$ are the same, the typical correlation time $\tau_{0}$ corresponding to $C_{0}$ is much larger than the correlation time $\tau_{0}\left(\ell /\left|\mathbf{r}-\mathbf{r}_{0}\right|\right)^{2}$ corresponding to $C_{1}$, as $\left|\mathbf{r}-\mathbf{r}_{0}\right| \gg \ell$. This stems from the fact that the decay of $C_{1}$ is due to multiple scattering events, each giving a contribution to the decorrelation process. The factor $\left|\mathbf{r}-\mathbf{r}_{0}\right| / \ell$ in Eq. (21) reflects the increase of the effective number of scattering events with $\left|\mathbf{r}-\mathbf{r}_{0}\right|$. In contrast, the decay of $C_{0}$ with $\tau$ originates from single scattering events in the neighborhood of the source. The following propagation of waves to the distant points $\mathbf{r}$ and $\mathbf{r}+\Delta \mathbf{r}$ depicted by the ladder propagators in Fig. $\mathbb{1}$, does not lead to any additional decorrelation, and thus the characteristic decay time of $C_{0}(\mathbf{r}, \Delta \mathbf{r}, \tau)$ is insensitive to $\mathbf{r}-\mathbf{r}_{0}$ and $\mathbf{r}+\Delta \mathbf{r}-\mathbf{r}_{0}$.

It is worthwhile to note that there exists another important difference between the $C_{0}$ and $C_{1}$ correlation functions. Eq. (21) for $C_{1}$ is derived under assumption of statistically homogeneous scatterer dynamics throughout the whole space [the characteristic time $\tau_{0}$ in Eq. (21) is assumed to be the same for all parts of the medium]. If, for instance, the scatterer motion in some region of space differs from that in the rest of the medium, the difference is reflected directly on the decay of $C_{1}$. This property of $C_{1}$ allows its use for imaging of scatterer dynamics in turbid media15. Moreover, space-resolved measurements of $C_{1}$ permit to distinguish between Brownian and directed (flow) motion of scatterers inside some bounded region of space, and to image scatterer flows in multiple-scattering media.16 In contrast, as the decay of $C_{0}$ is only sensitive to the motion of scatterers in the vicinity of the source, any modifications of scatterer dynamics in other parts of the medium do not affect the $C_{0}$ correlation [the characteristic time $\tau_{0}$ in Eqs. (18), (20) is that corresponding 
to the immediate neighborhood of the source]. The $C_{0}$ correlation function is not therefore suitable for imaging of the bulk of disordered media. As a compensation of this drawback, $C_{0}$ has, however, an important advantage as compared to $C_{1}$. The advantage is the spatiallyselective sensitivity to the scatterer motion in the immediate neighborhood of the source. This sensitivity opens up a possibility of "dynamic diffuse microscopy" of scatterer motion in multiple-scattering media.

The presented treatment of the space-time correlation is valid for $\Delta r>\ell$. If $\Delta r<$ $\ell$, an additional contribution to the correlation function appears due to scattering in the neighborhood of the observation points, just like in the case of purely spatial correlation. For $\Delta r=0$, the sum of the two contributions yields a correction to the $C_{1}$ correlation function [Eq. (21)]. A possible existence of such a correction has been suggested by Scheffold et al. 13 although they considered a different experimental geometry. This correction is uniquely determined by the motion of scatterers in the neighborhoods of the source and the observation point. We emphasize that $C_{0} \ll C_{1}$ for $\Delta r=0$, while for $\Delta r>\ell$ the $C_{1}$ term disappears and the leading contribution to the correlation function is given by $C_{0}$, since $C_{2} \sim\left(k_{0} \ell\right)^{-2} \ll C_{0}$.

\section{CONCLUSION}

In the present paper, we consider a recently suggested 6 novel type of intensity correlation in disordered media $\left(C_{0}\right)$. We show that the $C_{0}$ correlation function exhibits a strongly nonuniversal behavior in the sense that it is sensitive to the short-distance properties of disorder and geometrical parameters (e.g., size) of the localized source of waves. In particular, we find that $C_{0}$ depends explicitly on the correlation length of dielectric function fluctuations $\ell_{\varepsilon}$ and the size of the source $a$. More precisely, the $C_{0}$ correlation is damped down as either $\ell_{\varepsilon}$ or $a$ is

increased. As a function of $k_{0} \ell_{\varepsilon}, C_{0}$ exhibits linear decay for $k_{0} \ell_{\varepsilon} \ll 1$. As a function of $k_{0} a$, the $C_{0}$ correlation function also decays linearly for $k_{0} a \ll 1$, and $C_{0} \propto\left(k_{0} a\right)^{-1}$ for $k_{0} a \gg 1$. The sensitivity of the $C_{0}$ correlation to the short-distance properties of disorder (e.g., to $\ell_{\varepsilon}$ ) 
is its distinctive feature, since all other types of correlation commonly considered for waves in disordered media $\left(C_{1}, C_{2}, C_{3}\right)$ are insensitive to the details of scattering medium, and only depend on the mean free path, which is an integral parameter of disorder.

Next, we calculate the space-time correlation function of intensity fluctuations $C_{0}(\mathbf{r}, \Delta \mathbf{r}, \tau)$. We show that this correlation function is only sensitive to the scatterer motion in the immediate neighborhood of the source, while being insensitive to the dynamics of the rest of disordered medium. $C_{0}(\mathbf{r}, \Delta \mathbf{r}, \tau)$ is independent of $\mathbf{r}, \Delta \mathbf{r}$ and decays linearly as

a function of $\sqrt{\tau / \tau_{0}}$ for $\tau \ll \tau_{0}\left(\tau_{0}\right.$ is the single-scattering correlation time). For large $\tau$ $\left(\tau \gg \tau_{0}\right)$, the decays of $C_{0}$ is power-law: $C_{0} \propto\left(\tau / \tau_{0}\right)^{-3 / 2}$.

Finally, we suggest that the spatially-selective sensitivity of the $C_{0}$ correlation function to the properties and dynamics of disordered medium in the neighborhood of the source (and detector for $\Delta r<\ell$ ) may be used to develop a sort of "diffuse microscopy" of multiplescattering media.

\section{ACKNOWLEDGMENTS}

We would like to thank B. A. van Tiggelen for many stimulating discussions.

${ }^{1}$ M. C. W. van Rossum and Th. M. Nieuwenhuizen, Rev. Mod. Phys. 71, 313 (1999).

${ }^{2}$ S. Feng, C. Kane, P. A. Lee, and A. D. Stone, Phys. Rev. Lett 61, 834 (1988).

${ }^{3}$ R. Berkovits and S. Feng, Phys. Rep. 238, 135 (1994).

${ }^{4}$ C. P. Umbach, S. Washburn, R. B. Laibowitz, and R. A. Webb, Phys. Rev. B 30, 4048 (1984);

P. A. Lee and A. D. Stone, Phys. Rev. Lett. 55, 1622 (1985).

${ }^{5}$ F. Scheffold and G. Maret, Phys. Rev. Lett. 81, 5800 (1998).

${ }^{6}$ B. Shapiro, Phys. Rev. Lett. 83, 4733 (1999). 
${ }^{7}$ This is one of the possible and, perhaps, the simplest way to treat the case of the source of non-zero size. We expect that the final result is not very sensitive to the exact form of boundary conditions at $\left|\mathbf{r}-\mathbf{r}_{0}\right|=a$. An essential feature of our model of the source is that no scattering occurs at $\left|\mathbf{r}-\mathbf{r}_{0}\right|<a$.

${ }^{8}$ U. Frisch, in Probabilistic Methods in Applied Mathematics, edited by A. T. Bharucha-Reid (Academic, New York, 1968); S. M. Rytov, Yu. A. Kravtsov, and V. I. Tatarskii, Principles of Statistical Radiophysics (Springer-Verlag, New York, 1989).

${ }^{9}$ B. Shapiro, Phys. Rev. Lett. 57, 2168 (1986).

10 To carry out the integrations, we have used the formulas from I. S. Gradshteyn and I. M. Ryzhik, Tables of Integrals, Series and Products (Academic, Boston, 1994); A. P. Prudnikov, Yu. A. Brychkov, and O. I. Marichev, Integrals and Series (Gordon and Breach, New York, 1961).

${ }^{11}$ G. Maret and P. E. Wolf, Z. Phys. B 65, 409 (1987); M. Rosenbluh, M. Hoshen, I. Freund, and M. Kaveh, Phys. Rev. Lett. 58, 2754 (1987); D. J. Pine, D. A. Weitz, P. M. Chaikin, and E. Herbolzheimer, Phys. Rev. Lett. 60, 1134 (1988).

12 M. J. Stephen, Phys. Rev. B 37, 1 (1988).

${ }^{13}$ F. Scheffold, W. Härtl, G. Maret, and E. Matijević, Phys. Rev. B 56, 10942 (1997).

${ }^{14}$ A. A. Golubentsev, Sov. Phys. - JETP 59, 26 (1984).

${ }^{15}$ D. A. Boas, L. E. Campbell, and A. G. Yodh, Phys. Rev. Lett. 75, 1855 (1995); D. A. Boas and A. G. Yodh, J. Opt. Soc. Am. A 14, 192 (1997).

${ }^{16}$ M. Heckmeier and G. Maret, Europhys. Lett. 34, 257 (1996); M. Heckmeier, S. E. Skipetrov, G. Maret, and R. Maynard, J. Opt. Soc. Am. A 14, 185 (1997); S. E. Skipetrov and I. V. Meglinskiü, JETP 86, 661 (1998). 


\section{FIGURE CAPTIONS}

FIG. 1. The leading diagram contributing to the correlation function $\langle\delta I(\mathbf{r}, t) \delta I(\mathbf{r}+\Delta \mathbf{r}, t+\tau)\rangle$. $L$ 's denote the ladder propagators, the left propagator corresponds to time $t$, and the right one to $t+\tau$. Solid lines with arrows denote retarded (with arrows directed from $\mathbf{r}_{0}$ to $\mathbf{r}$ or $\mathbf{r}+\Delta \mathbf{r}$ ) and advanced (with arrows directed from $\mathbf{r}$ or $\mathbf{r}+\Delta \mathbf{r}$ to $\mathbf{r}_{0}$ ) average Green functions, $\bar{G}$ and $\bar{G}^{*}$, respectively. $V$ is the vertex located at $\mathbf{r}_{0}$. Diagrams contributing to $V$ are shown in Fig. 2 .

FIG. 2. The diagrams contributing to the vertex $V$. Solid lines with arrows directed from $\mathbf{r}_{0}$ to some other point denote retarded average Green functions $\bar{G}$, solid lines with arrows directed to $\mathbf{r}_{0}$ denote advanced average Green functions $\bar{G}^{*}$. In each diagram, the left pair of Green functions corresponds to time $t$, and the right pair - to $t+\tau$. Crosses indicate scattering (a factor $k_{0}^{2}$ is associated with each cross), dashed lines denote correlation function $B_{\varepsilon}(\Delta \mathbf{r}, \tau)$ of the fluctuating part of the dielectric constant. Integrations are assumed over $\mathbf{r}_{1}, \mathbf{r}_{2}, \mathbf{r}_{3}$, and $\mathbf{r}_{4} \cdot \mathbf{r}_{0}$ is the source position.

FIG. 3. Function $F(\alpha, \beta)$ describing the effects of non-zero correlation length $\ell_{\varepsilon}$, size of the source $a$, and time delay $\tau$ on the $C_{0}$ correlation function. The solid line is $F(\alpha, 0)$, and the dashed line is $F(0, \beta)$. The attenuation factor which multiplies the $C_{0}$ value found for $\ell_{\varepsilon}=a=0$ and $\tau=0$

is obtained by setting $\alpha=k_{0} \ell_{\varepsilon}$ (disorder with non-zero correlation length, $\left.k_{0} \ell_{\varepsilon} \ll 1\right), \alpha=\left(\tau / \tau_{0}\right)^{1 / 2}$ (scatterers undergoing Brownian motion), or $\beta=k_{0} a$ (non-zero size of the source). 


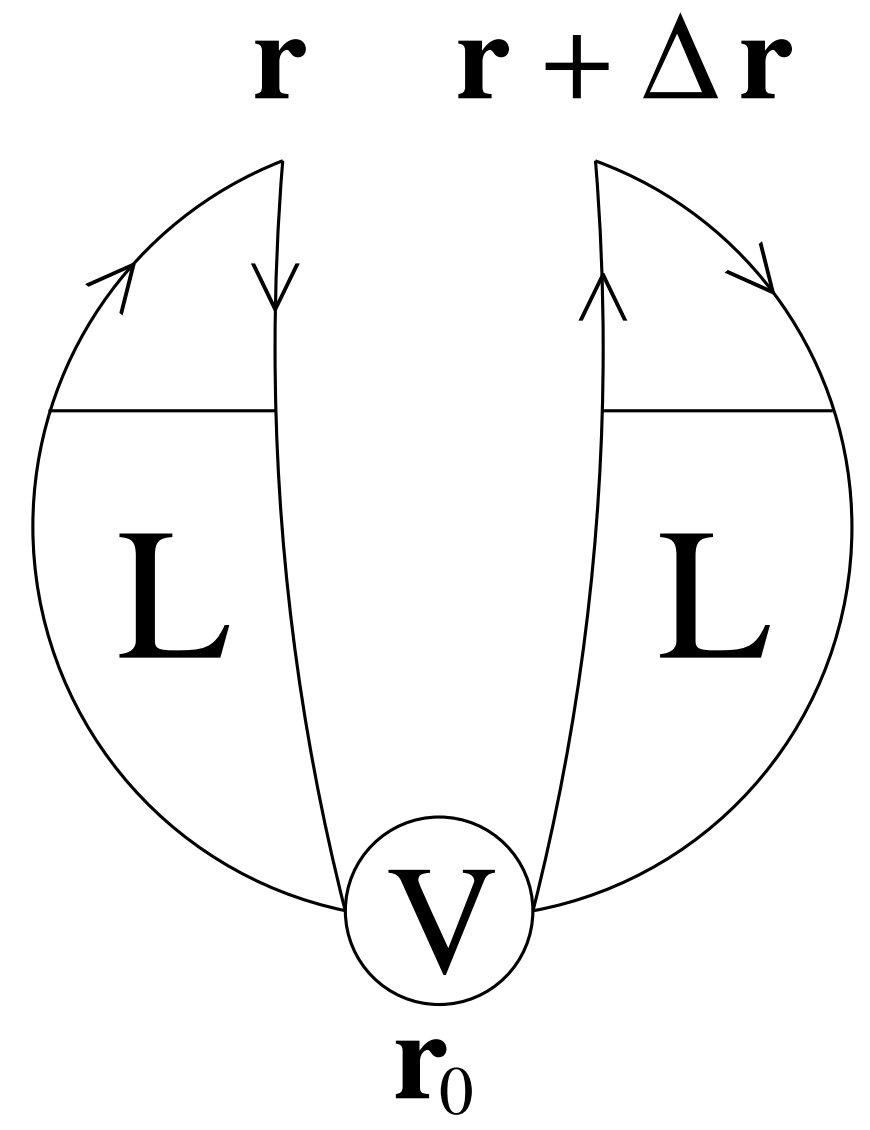




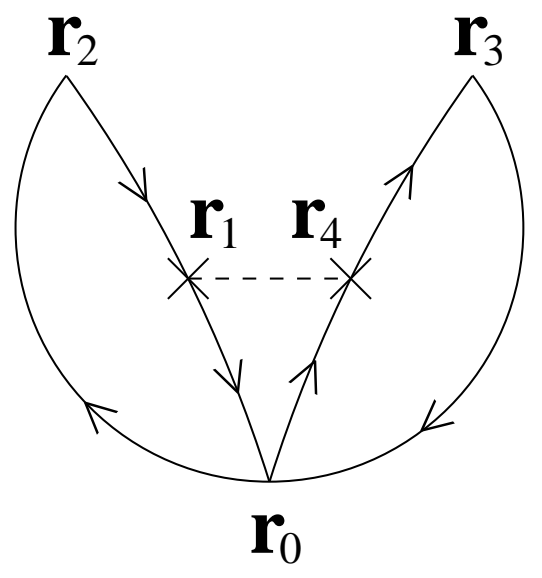

(a)

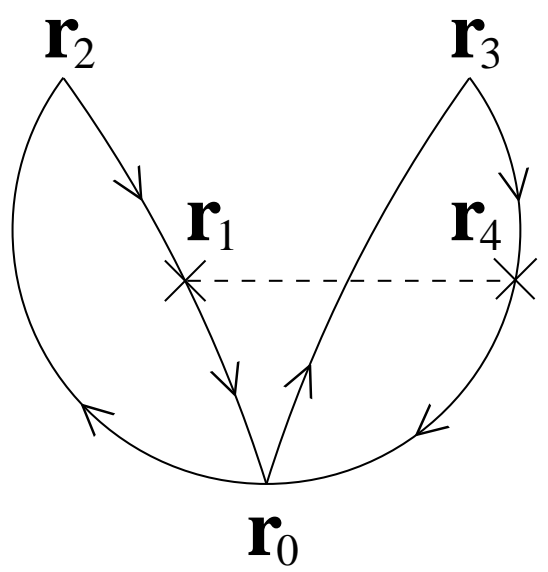

(c)

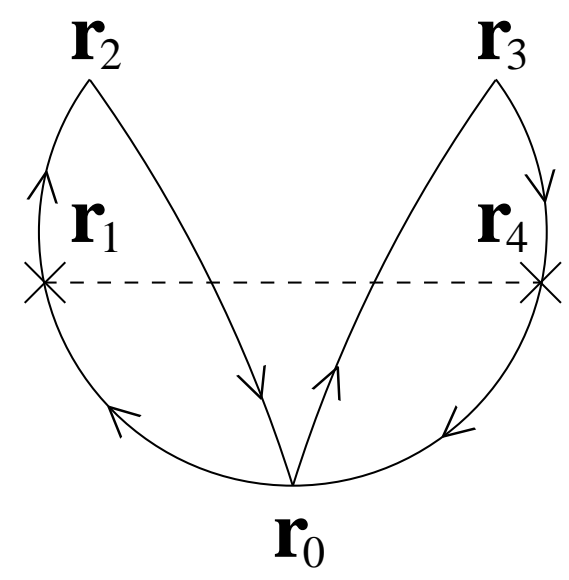

(b)

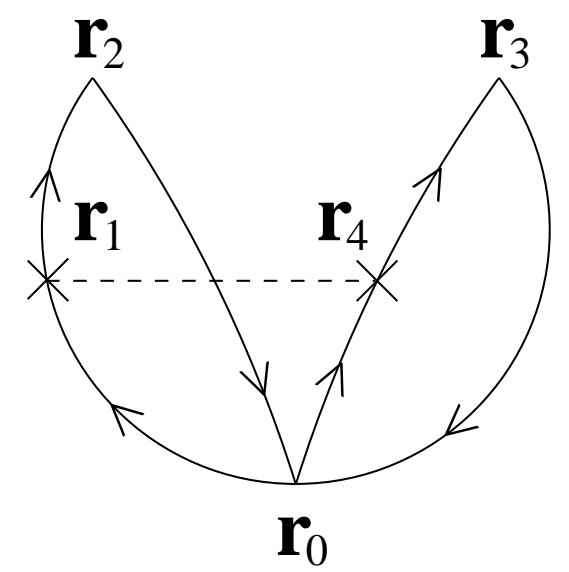

(d) 


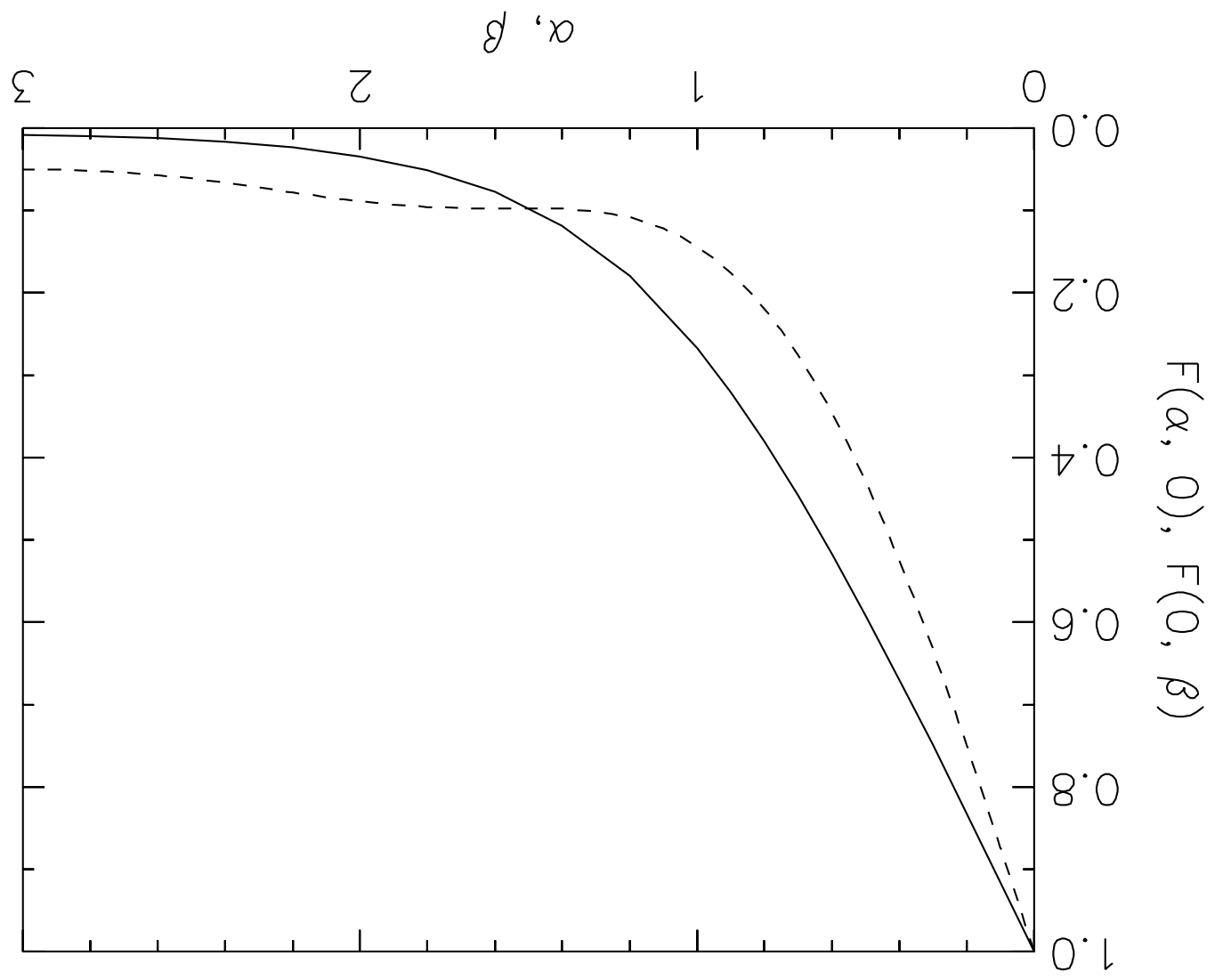

